patients on PN who have not received NST or dietitian intervention during the period from Mar 2016 - Mar 2017. Energy, protein, and micro-nutrients consumption adequacy were compared between the intervention and control groups. Patients without body weight and nutrition intake records were excluded. Independent t-test and chi-square test were used to compare the variables between both groups. A p-value of $<0.05$ was considered statistically significant.

Results The patients in the intervention group had a significantly higher energy and protein intake than that of the control group $(1279 \pm 353 \mathrm{kcal}$ versus $934 \pm 261 \mathrm{kcal} ; 58 \pm 16 \mathrm{~g}$ versus $43 \pm 12 \mathrm{~g}$ ). In terms of nutritional intake adequacy, the percentage of targeted energy and protein requirement achieved is significantly higher in the intervention group (81 $\pm 21 \%$ and $90 \pm 28 \%$ of their energy and protein requirements respectively), compared to $64 \pm 21 \%$ and $75 \pm 23 \%$ in the control group. Adequacy of micronutrient consumption is also significantly higher in the intervention group, $88.6 \%$ of patients in the intervention group achieved adequate intake of micronutrients from PN compare to $8.1 \%$ of patients in the control group.

Conclusions Nutrition care provided by the NST is promising to ensure the nutrition well-being of hospitalized malnourished patients. This review suggests that NST intervention can improve both macro- and micronutrient intake adequacies in patients on parenteral nutrition support. Further clinical outcomes studies on the benefits of NST are recommended.

\section{IDDF2021-ABS-0011 ENDOSCOPIC ULTRASONOGRAPHY IS USEFUL IN PREDICTING PERFORATION OF ENDOSCOPIC RESECTION FOR GASTRIC SUBMUCOSAL TUMORS ORIGINATING FROM THE MUSCULARIS PROPRIA}

Yi Lu*, Xianhua Zhuo, Jiachen Sun, Chujun Li. The Sixth Affiliated Hospital, Sun Yat-sen University, China

\subsection{6/gutjnl-2021-IDDF.105}

Background Predicting the risk of perforation during the endoscopic resection (ER) of gastric submucosal tumors (SMTs) originating from the muscularis propria (MP) could remind us to be more cautious and be prepared ahead of time. Moreover, we could select the appropriate patients for the inexperienced endoscopists. But for now, the predictive model was rare. Therefore, we conducted this study to figure out important parameters in the endoscopic ultrasonography (EUS) images to predict perforation, and try to build predictive models.

Methods From May 1st, 2013, to January 15th,2021, consecutive patients with gastric SMTs originating from MP and received ER in our hospital were retrospectively reviewed. The patients were classified into two groups based on whether they had perforation (the case group) or without (the control group). To evaluate the depth of the SMT locates using the quantification index, we came up with a parameter called the ratio of inner distance to outer distance (I/O ratio). Logistic multivariate analysis was used to identify potential variables and predictive models.

Results 199 EUS procedures (194 patients) were finally chosen for analysis. The median age of the patients was 53 years old, and $88(44.22 \%)$ were males. The en bloc resection rate had no difference in the two groups, while patients in the case group had longer procedure time, higher probability of infection, and longer postoperative hospital stay. Multivariate analysis showed that age, I/O ratio, and the pathology of the tumors were risk factors for perforation, and we further built two models to predict perforation, with an AUC of 0.836 (for Model 1) and 0.755 (for Model 2).

Conclusions EUS was useful in predicting perforation of ER for gastric SMTs originating from MP, and we have identified two models to predict perforation, which might serve as a useful tool in clinical practice.

\section{IDDF2021-ABS-0016 EFFICACY AND SAFETY OF PRE- ENDOSCOPIC TREATMENT FOR ASYMPTOMATIC OR INCIDENTAL STRICTURES IN CROHN'S DISEASE}

Junrong Chen*, Jiachen Sun, Yi Lu, Min Zhi. The Sixth Affiliated Hospital, Sun Yat-sen University, China

\subsection{6/gutjnl-2021-IDDF.106}

Background Current important treatment modality for stricture in Crohn's disease (CD) is endoscopic treatment. However, there is no unified treatment plan for asymptomatic or incidental strictures with CD patients (AIS).

Methods All eligible patients with stenosis of CD who were treated by endoscopic therapy from January 2013 to January 2021 were included (IDDF2021-ABS-0016 Figure 1. Endoscopic balloon dilation of small intestinal stricture with CD patients. A, Small intestinal stricture; B, Place $35 \mathrm{GW}$ through the stenosis segment; C, Dilation with $16 \mathrm{~mm}$ through-the-scope balloon; D, Post-dilation appearance). The primary outcomes were treatment success rate, post-procedural complications, endoscopic recurrence, additional surgical treatment rate, and stenosis-free survival with preendoscopic treatment for AIS or endoscopic treatment for symptomatic strictures.

Results A total of 87 patients of 125 procedures were studied, including pre-endoscopic treatment group $(\mathrm{N}=31)$ and endoscopic treatment group $(\mathrm{N}=56)$. The median follow-up time was 21 months (2-91 months). Cox multivariate regression analysis found that the type of treatment (HR 3.146, 95\%CI 1.081-9.156, $\mathrm{P}=0.036)$, balloon diameter (HR 3.877, 95\%CI 1.343-6.466, $\mathrm{P}=0.009$ ), total enteral nutrition (HR 2.946, 95\%CI 1.112-11.323, $\mathrm{P}=0.007$ ), and history of anal fistula surgery (HR 3.548, 95\%CI 1.412 10.648, $\mathrm{P}=0.032$ ) were risk factors for recurrent stenosis after endoscopic treatment. While duration (HR 5.860, 95\% CI 1.068-32.163, $\mathrm{P}=0.042$ ) and hypoproteinemia (HR 4.578 , 95\%CI $1.038-20.196, \mathrm{P}=0.045)$ were risk factors for additional surgery after endoscopic treatment. Compared with endoscopic treatment group, pre-endoscopic treatment group had the immediate technical success for therapy fairly (96.77\% vs. 91.07\%, P=0.573), lower endoscopic stenosis recurrence rate $(16.13 \%$ vs. $46.43 \%, \mathrm{P}=0.003)$, lower surgical treatment rate ( 0 vs. $16.07 \%, P=0.024)$, and higher 3 years stenosis-free survival $(85.9 \%$ vs. $50.4 \%)$. The postoperative complications in all 3 cases, bleeding in 1 case, 2 cases of perforation were in the endoscopic treatment group.

Conclusions CD patients with AIS were often inadvertently found during colonoscopy and might develop symptomatic 


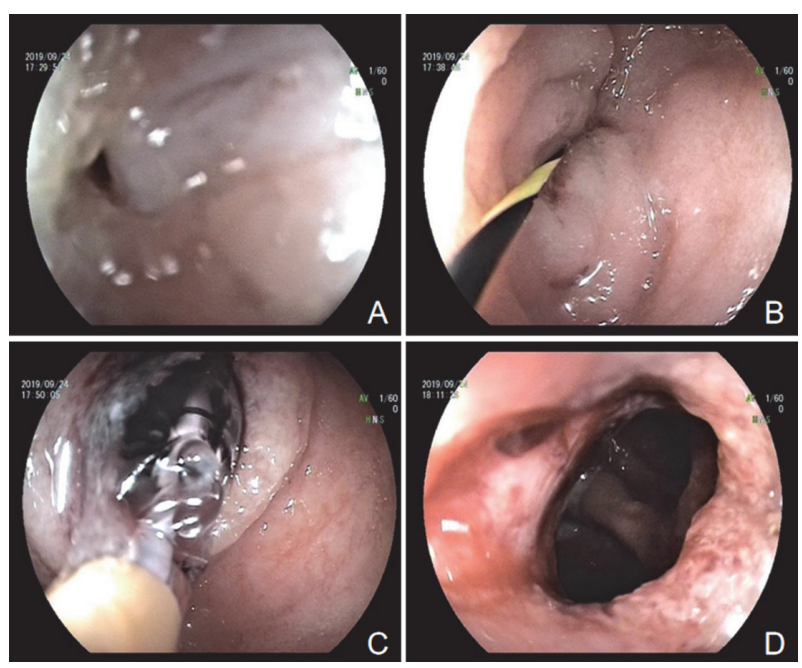

Abstract IDDF2021-ABS-0016 Figure 1

stenosis in the future. Pre-endoscopic treatment for AIS has high safety and definite short-term efficacy, which can improve the prognosis and the quality of life of patients. The longterm effect needs to be further followed up.

\section{IDDF2021-ABS-0017 DEVELOPMENT AND VALIDATION OF A NEW ALGORITHM MODEL FOR DIFFERENTIAL DIAGNOSIS BETWEEN CROHN'S DISEASE AND INTESTINAL TUBERCULOSIS}

Yi Lu*, Jiayin Yao, Min Zhi. The Sixth Affiliated Hospital, Sun Yat-sen University, China

\subsection{6/gutjnl-2021-IDDF.107}

Background Sometimes it was a great challenge to distinguish Crohn's disease (CD) and intestinal tuberculosis (ITB); we conducted this study was to identify a simple and useful algorithm for distinguishing them.

Methods We retrospectively reviewed the medical history of the patients who were diagnosed as ITB or CD. We firstly identified ITB patients, and then the patients diagnosed with CD were matched by age, sex, and admission time in a 1:1 ratio. Patients who were admitted between May 1, 2013 and April 30, 2019 were regarded as training cohort, and patients admitted between May 1, 2019 and May 1, 2020 were regarded as validation cohort. We used multivariate analysis to identify the potential variables, and then we used $\mathrm{R}$ package part to build the classification and regression tree (CART), and validated the newly developed model.

Results In total, the training cohort included 84 ITB and 84 CD patients, and the validation cohort included 22 ITB and $22 \mathrm{CD}$ patients. Multivariate analysis showed that positive TSPOT, $\geq 4$ segments involved, longitudinal ulcer, circular ulcer, and aphthous ulcer, were confirmed as independent discriminating factors. Using these parameters to build the CART model made an overall accuracy rate was $88.64 \%$, with sensitivity, specificity, NPV, and PPV being 90.91\%, 86.36\%, $90.48 \%$, and $86.96 \%$, respectively.

Conclusions We developed a simple and novel algorithm model covering laboratory, imaging, and endoscopy parameters with CART to differentiate ITB and CD with good accuracy.

\section{IDDF2021-ABS-0020 A SURVIVAL ANALYSIS OF CHINESE PATIENTS WITH GASTROINTESTINAL MUCOSAL MELANOMAS: A SINGLE- CENTER RETROSPECTIVE STUDY}

Junrong Chen*, Jiachen Sun, Jiayao Huang. The Sixth Affiliated Hospital, Sun Yat-sen University, China

\subsection{6/gutjnl-2021-IDDF.108}

Background Mucosal melanomas are highly malignant tumors, while are of great interest due to their aggressive behavior and less favorable prognosis, which could be the result of many reasons, for example, late diagnosis, patients' delay and the obscured anatomic site of origin. We, in this paper, do a retrospective study to investigate the characteristics and prognosis of gastrointestinal mucosal melanomas in a Chinese population to help future clinic doctors to recognize the prognosis of the disease.

Methods We retrospectively studied 49 patients diagnosed with gastrointestinal mucosal melanomas. Multivariate analyses of

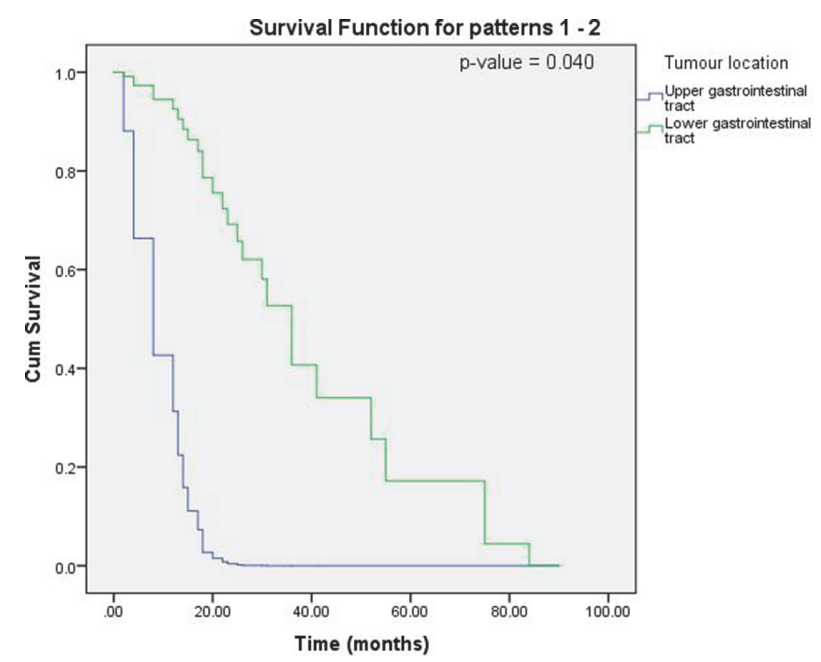

Abstract IDDF2021-ABS-0020 Figure 1

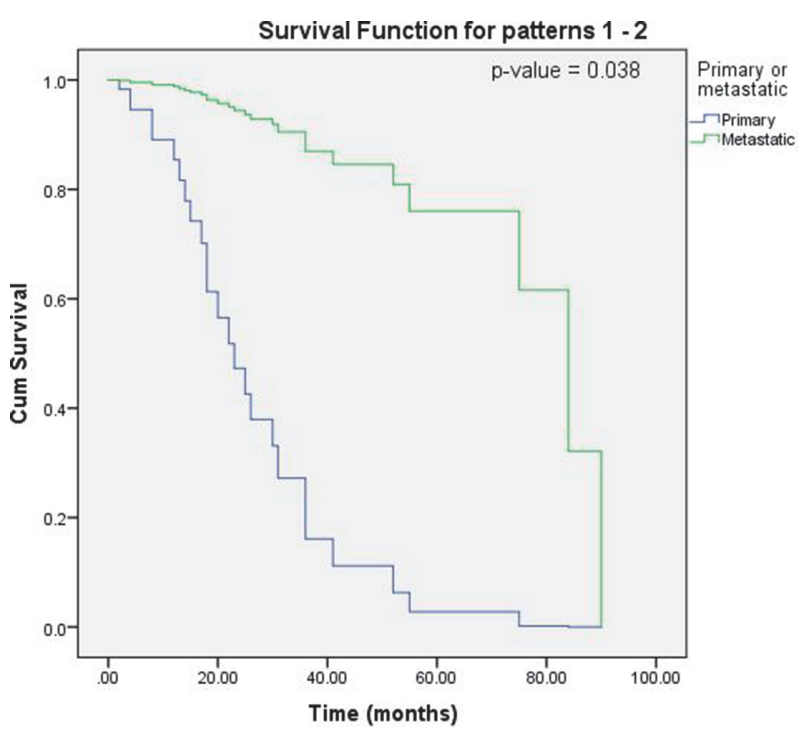

Abstract IDDF2021-ABS-0020 Figure 2 